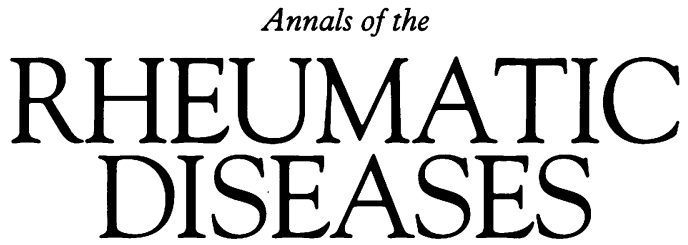

Leader

\title{
The significance of vascular inflammation in rheumatoid arthritis
}

\section{The clinical spectrum and incidence of vascular involvement in rheumatoid arthritis}

Vasculitis is an aspect of systemic rheumatoid arthritis (RA) that is viewed very differently by rheumatologists according to their background. Academic centres may see it as a major problem, with some enthusiasts even supporting Bywater's provocative suggestion that vasculitis is the basis of all systemic connective tissue disease. ${ }^{1}$ On the other hand, the busy clinician may feel that it is an infrequent, diminishing clinical problem, "so rare that it really does not rate attention". The truth probably lies between these two extremes, since in medicine, as in other aspects of life, we only see what we are looking for.

The first problem is in trying to establish the true frequency and extent of vascular involvement in RA. More data, such as that provided by Flipo et al in this issue, ${ }^{2}$ provides new insights into this. The second problem is in the assessment of the immediate implications of vascular inflammation when it is detected in RA. This is made complex by the wide spectrum of both the pathology and the clinical manifestations. Clinically apparent disease spans from frequent, apparently benign aspects such as, nail-edge lesions, through to rare but major features associated with a high mortality such as, mononeuritis multiplex. This clinical spectrum reflects in part the vessel size involved pathologically, ranging from arteriolar capillary through to major muscular artery. In individual patients, it is often difficult to obtain biopsy confirmation of the diagnosis despite clinically active disease. This provides the driving force to establish new and more sensitive tests, such as that described here by the French group, ${ }^{2}$ who suggest a high diagnostic yield from labial gland biopsy.

The third, and possibly most important problem is to assess the long term sequelae of vascular involvement in RA. It is of great interest that Flipo et al also record a significant yield of positive results in the control group of RA patients, with no apparent clinical disease correlates. This suggests the existence of widespread sub-clinical vasculitis. It is worth examining what the latter represents in terms of the spectrum of vasculitis and to ponder its real clinical significance. The purpose of this editorial is to suggest that vascular inflammation-whether overt or subclinical-is both a common feature of active RA and has broader clinical implications than is usually appreciated. We propose that it may be the major determinant of accelerated atherosclerosis and thus of the increased cardiovascular mortality noted in RA. ${ }^{3}$

\section{Small vessel disease}

Small vessel disease is widely viewed as a frequent but generally unimportant aspect of RA. Like other aspects of systemic RA, such as pericarditis, ${ }^{4}$ the recorded incidence reflects the perseverance which has been put into the observations. ${ }^{5}$ Small vessel vasculitis commonly involves the skin and the existence of a high incidence of subclinical disease is indicated by the ease with which trauma or active hand use may induce clinically apparent lesions, classically in 'typus robustus' RA. The common lesions at the nail fold or nail edge may even relate to simple local pressure, such as holding a pen. ${ }^{6}$ These clinical observations are amplified by the finding of small vessel vasculitis in biopsy of apparently uninvolved skin in $30 \%$ of RA patients. ${ }^{78}$ If trauma is the chief factor determining the site of overt lesions, there is no shortage of potential mechanisms which may underlie the general vascular pathology. The most obvious is immune complexmediated damage, the classic experimental model for vasculitis. Indeed certain types of large or insoluble complexes, particularly those containing IgG rheumatoid factors, are associated with overt major vessel vasculitis. ${ }^{9} 10$ Overall in active RA, however, the presence of circulating immune complexes, rheumatoid factors, and even evidence of complement consumption, is recorded far more commonly. ${ }^{11}$ The pathogenetic potential of these findings has not been properly assessed in the context of subclinical disease nor have they been related to biopsy findings.

\section{Vasculitis in muscular arteries}

Vasculitis involving larger vessels, such as small and medium muscular arteries, is by contrast widely accepted as an important part of the clinical pathology of RA. This produces the type of systemic necrotising vasculitis which has been likened to the development of PAN on a background of RA. ${ }^{12}$ It has been suggested that such systemic rheumatoid vasculitis is becoming less frequent, and may even be a disappearing disease. However, detailed studies over six years in Norwich have for the first time established an actual incidence figure $\left(1.3\right.$ per $10^{5}$ 
population) which is higher than previous estimates. ${ }^{13}$ Rheumatoid vasculitis was at first only recognised clinically when presenting as major lesions such as mononeuritis multiplex or gangrene. ${ }^{14}{ }^{15}$ Increased interest both added to the number of cases reported and broadened the spectrum of clinical disease recognised. ${ }^{16}$ In particular, the importance of nonspecific systemic manifestations such as weight loss and malaise, as well as cutaneous manifestations, became apparent. From there, it is only a small step to recognising subclinical vascular inflammation as an important factor to exclude when unexplained weight loss occurs in RA-a not uncommon event.

An active policy of obtaining biopsy material (to be examined by a dedicated pathologist) reveals a further incidence of definite, active necrotising vasculitis in cases without any overt symptoms. ${ }^{17}$ This may also be seen in routine histology of surgical specimens from RA patients, raising currently unanswerable questions regarding the attribution of the original surgical presentation. Thus it appears that even necrotising vascular inflammation can be sub-clinical. There is an analogy with the well described 'localised PAN'. Many authors have doubted whether that process is indeed truly localised, ${ }^{18}$ while the mechanisms which could contain what appears to be a systemic process to a localised set of blood vessels are completely obscure. It is equally possible that vascular inflammation is much more widespread, even at first presentation, than the initial clinical symptoms and signs indicate.

\section{Significance of subclinical vasculitis}

It thus appears that sub-clinical vascular inflammation, involving all sizes of afferent vessels, may be common in $\mathrm{RA}$ - as in other diffuse non-organ specific autoimmune diseases, such as SLE. The significance of these findings needs to be examined from the short and longer term viewpoints. The initial discovery often provokes considerable alarm, even if it is incidental to other pathology and completely unexpected. The first reaction may be to take panic measures and institute aggressive therapy. The cool response of the experienced vasculitis expert may be to observe and do nothing in these cases, on the grounds that such patients often come to no harm and therapy is more appropriately directed to clinical disease than to tests. In the long term, neither of these approaches may be entirely correct.

In the short term, sub-clinical vasculitis almost certainly makes a significant contribution to the systemic illness causing malaise, fever and weight loss that characterise the active phases of RA. Such constitutional symptoms are very suggestive of an underlying vasculitis ${ }^{19}$ and may be due to systemic production of cytokines such as IL-1, IL-6 and TNF which in addition to inducing the acute phase response can also affect endothelial cell function, ${ }^{20}$ integrating them in the overall immunological phenomena observed in RA. Thus vasculitis, even when 'subclinical', may be driven by mechanisms intrinsic to other RA pathology, influence the course of the disease and make a direct contribution to the immediate clinical picture.

In the long term sub-clinical vasculitis has the potential to make a further important contribution to the morbidity and the accelerated mortality observed in long standing RA. The late consequences of vascular inflammation are increasingly being recognised as being far from benign, even when healing has occurred. They present as accelerated or widespread 'degenerative' vascular disease. In RA, medial degeneration and vascular calcification is well documented. ${ }^{21}$ Despite the suggestion that widespread vasculitis and transformation to occlusive vasculopathy is due to chronic steroid therapy, ${ }^{22}$ patients who have never received steroids do develop vasculitis. ${ }^{23}$ It is more likely that the clinically overt lesions that may follow an abrupt change in steroid dose result from uncovering a preexisting sub-clinical vascular involvement. In SLE there is also an increase in early atheroma ${ }^{24}$ not directly linked to steroid therapy here or in RA. ${ }^{25}$ The extent of such late effects of vascular inflammation is currently unknown.

We propose that vascular involvement in RA, whether overt or sub-clinical, has a major role in the well documented but currently unexplained increased mortality of RA, particularly the excess mortality related to cardiovascular disease. ${ }^{3}$ If this hypothesis is correct, subclinical vascular inflammation needs to be taken far more seriously than at present. More accurate data on the frequency and distribution of blood vessel inflammation would be very useful clinically, as well as being of academic interest. The paper by Flipo et al makes a real contribution here, by offering further insight into the widespread nature of such involvement in RA and by suggesting a new, highly accessible site to study incidence during the acute stage. Unfortunately, its value is somewhat limited by the extreme rarity of muscular arteries in such biopsies, so that only the presence of small vessel disease can be assessed at this site. However, it does support the overall thesis that there is more sub-clinical vasculitis in RA than is generally appreciated. Rectal biopsy, where muscular arteries are frequently observed in the deeper layers, may further increase the useful information base.

\section{Mechanisms of chronic vascular damage}

The concept that the immunological mechanisms inducing vascular inflammation can also be the precursor of chronic vascular damage is entirely consistent with the response to injury hypothesis, currently one of the major theories for the genesis of atheroma. ${ }^{26}$ Injury to endothelium, which occurs in vasculitis, is the key initiating event in this hypothesis. It is well known that experimental autoimmunity can induce rampant atheroma, at least in rabbits. ${ }^{27}$ Atheroma itself may have an immune element, since the lesions contain CD4+ and CD8+ lymphocytes as well as macrophages. ${ }^{28} 29$ This concept also agrees with the observations in other fields of immunopathology, particularly recent studies in graft rejection. In chronic graft rejection the most significant pathological finding is a form of accelerated arteriosclerosis showing diffuse intimal proliferation involving large or medium arteries as well as arterioles. ${ }^{30}$ This is very reminiscent of the "bland digital vasculitis' described by Bywaters in chronic RA. ${ }^{15}$ The early development and high incidence of such lesions in cardiac grafts reflects the particular sensitivity of the myocardium, with its strict dependance on the coronary blood flow. Currently, vascular disease is the leading cause of death or graft failure in cardiac transplants, occurring in more than $80 \%$ of those surviving five years. ${ }^{31}$ Such vascular problems are by no means confined to cardiac grafts, being well described in renal, hepatic, and lung grafts. The mechanism for such arteriosclerotic lesions is the subject of intense current research effort. Studies in rats suggest that $100 \%$ of allografts develop similar lesions, even in transplants between MHC identical strains that differ at other loci. The early lesions are sub-endothelial accumulations of mononuclear cells. The late picture is one of hypo-cellular lesions, except for occasional focal infiltrates, but with diffuse intimal thickening. We have seen a similar picture develop in serial biopsy as necrotising vasculitis heals in RA. This suggests that early inflammatory cell/endothelial cell interactions, with local 
cytokine production, is responsible for the development of the late arteriosclerotic lesions. The most important cell is probably the monocyte which adheres to injured endothelial cells with up-regulated MHC class II and ICAM-1 expression. ${ }^{32}$ Under the influence of other cytokines and growth factors, such as $\gamma$-IFN and PDGF, monocytes accumulate and secrete their own damaging factors. These include MCP-1 and induce subsequent smooth muscle proliferation plus extra-cellular matrix formation. ${ }^{33}$

The importance of elucidating these mechanisms in detail is that this will open the way to new therapeutic manoeuvres, which may be as relevant in RA as in graft rejection. Current therapy is unsatisfactory. It is already clear that inhibition of prostanoids cannot prevent the development of the experimental lesions, while immunosuppressive therapy may even accelerate them. ${ }^{34}$ This is consistent with our observations in human necrotising vasculitis that aggressive immunosuppressive regimes, although very effective in inducing remission, are not so good at preventing late manifestations of disease. ${ }^{35}$ Thus possible novel therapies are being studied with great interest.

\section{Conclusion}

Clinical observations and pathological findings support the concept of widespread sub-clinical vascular involvement in $\mathrm{RA}$, which in turn may have an impact in the longer term on the increased cardiovascular morbidity and mortality observed in this disease. The significance of positive biopsies, unrelated to clinical lesions, in active RA needs to be rigorously investigated. It will be important to document the incidence, frequency, and persistence of such subclinical vascular inflammation. In the longer term, its temporal and spatial relationship to chronic vasculopathy and cardiovascular morbidity must be assessed. That will require new investigative methods, since biopsy alone can only provide a single frame where we need the complete film. The importance of biopsy is that it frequently does throw up unexpected results which do not fit current concepts of vasculitis as an acute, often life threatening set of syndromes. We suggest that rheumatologists need to focus on this area as a major, clinically relevant problem.

Department of Rheumatology,

P A BACON

University of Birmingham,

Edgbaston, Birmingham,

United Kingdom

1 Bywaters E G L. Vasculitis in rheumatoid arthritis. In: Feltkamp T E W, ed. Non-articular forms of rheumatoid arthritis, Proc IV ISRA symposium, Leiden. Staffleu Sci 1976; 82-4.

2 Flipo R, Janin A, Hachulla E, et al. Labial salivary gland biopsy assessment in rheumatoid vasculitis. Ann Rheum Dis 1994; 53: 648-52.

3 Symmons D P M. Mortality in rheumatoid arthritis. Br $\mathcal{F}$ Rheum 1988; 27: 44-54. 4 Kirk J, Cosh J. The pericarditis of rheumatoid arthritis. Qf Med 1969; 152:
397-423.

5 Gordon D A, Stein J L, Broder I. The extra-articular features of rheumatoid arthritis. A systematic analysis of 127 cases. Am $\mathcal{F}$ Med 1973; 54: 445-52.

6 Edwards J C W. Relationship between pressure and digital vasculitis in rheumatoid disease. Ann Rheum Dis 1980; 39: 138-40.

7 Wested M L, Meijer C J L, Vermeer B J, Cats A, de Vries E. Rheumatoid arthritis-the clinical significance of histo and immunopathological abnormalities in normal skin. 1985; 11: 448-53.

8 Fitzgerald O M, Barnes L, Woods R, McHugh L, Barry C, O'Loughlin S. Direct immunofluorescence of normal skin in rheumatoid arthritis. $B \dot{j}$ Rheumatol 1985; 24: 340-5.

9 Elson C J, Scott D G I, Blake D R, Bacon P A, Holt P D J. Complement activating rheumatoid factor containing complexes in patients with rheumatoid vasculitis. Ann Rheum Dis 1983; 42: 147-50.

10 Pope R M, McDuffy S J. IgG rheumatoid factor: analysis of various species of IgG for the detection by radioimmunoassay. Arthritis Rheum 1980; 23. 733.

11 Scott D G I, Bacon P A, Allen C, Elson C J, Wallington T. IgG rheumatoid factor, complement and immune complexes in rheumatoid synovitis and vasculitis: comparative and serial studies during cytotoxic therapy. Clin Exp Immunol 1981; 43: 54-63.

12 Ball J. Rheumatoid arthritis and polyarteritis nodosa. Ann Rheum Dis 1954; 13: $277-90$.

13 Watts R A, Luqmani R A, Carruthers D M, et al. Systemic Rheumatoid Vasculitis (SRV)-A persistent problem. Clin Rheum 1994; 13: 371.

14 Schmidt F R, Cooper N S, Ziff M, McEwan C. Arteritis in rheumatoid arthritis. Am $\mathcal{F}$ Med 1961; 30: 56-83.

15 Bywaters E G L, Scott J T. The natural history of vascular lesions in rheumatoid arthritis. 7 Chron Dis 1963; 16: 905-14.

16 Scott D G I, Bacon P A, Tribe C R. Systemic rheumatoid vasculitis: a clinical and laboratory study of 50 cases. Medicine (Baltimore) 1981; 34: $843-50$.

17 Tribe C R, Scott D G I, Bacon P A. The place of rectal biopsy in the diagnosis of systemic vasculitis. $\mathcal{F}$ Clin Path 1981; 34: 843-50

18 Bacon P A. Systemic vasculitis syndromes. Current opinion in rheumatology, 1993; 5: 5-10.

19 Bacon P A. Extra-articular rheumatoid arthritis. In: McCarty D J, Koopman W J, eds. Arthritis and allied conditions, Vol 1, 12th ed. Philadelphia: Lea and Febiger, 1993: 811-40.

20 Bevilacqua M P. Endothelial-leukocyte adhesion molecules. Ann Rev Immunol 1993; 11: 767-804.

21 Forsyth $\mathrm{C}$ C. Calcification of the digital vessels in a child with rheumatoid arthritis. Arch Dis Child 1960; 35: 296.

22 Baggenstoss A H, Schick R M, Polley H F. The effects of cortisone on the lesions of periarteritis nodosa. Am ₹ Pathol 1951; 27: 537-43.

23 Kemper J W, Baggenstoss A H, Slocumb C H. The relationship of therapy with cortisone to the incidence of vascular lesions in RA. Ann Int Med 1957; 46: 831-9.

24 Ginzler E M, Schorn K. Mortality in sytemic lupus erythematosus. f Rheumatol 1987; 14: 218-22 (Suppl 13)

25 Raynauld J P, Wolfe F, Sibley J T, Fries J F. Mortality by cardiovascular disease and use of corticosteroids in rheumatoid arthritis. Arthritis Rheum 1993; 36: S193.

26 Ross R. The pathogenesis of atherosclerosis: a perspective for the 1990 s. Nature 1993; 362: 801-9.

27 Minick C R, Murphy G E. Experimental induction of atheroarteriosclerosis by the allergic injury to arteries and lipid-rich diets II. Effect of repeated injections of horse serum in rabbits fed lipid-rich, cholesterol-poor diet. Am $\mathcal{F}$ Pathol 1973; 73: 265-76.

28 Gown A M, Tscikada T, Ross R. Human atherosclerosis: II. Immunocytochemical analysis of the cellular composition of human atherosclerotic lesions $A m$ F Pathol 1986; 125: 191-9.

29 Emeson E E, Robertson A L. T Lymphocytes in aortic and coronary intimas. Their potential role in atherogenesis. Am f Pathol 1988; 130: 369-78.

30 Ewel C H, Foegh M L. Chronic Graft Rejection: Accelerated Transplant Arteriosclerosis. Immunol Reviews 1993; 134: 21-32.

31 Gao S Z, Schroeder J S, Hunt S, Stinson E B. Retransplantation for severe accelerated coronary disease in heart transplant recipients. Am $\mathcal{f}$ Cardiol $1988 ; 62: 867$.

32 Rosenheld M E, Faggioto A, Ross R. The role of the mononuclear phagocyte in primate and rabbit models of atherosclerosis. In: Van Furth R, ed: Mononuclear phagocytes: characteristics, physiology and function. The Hague, Netherlands: Martinus Nijhoff, 1985: 795.

33 Adams D H, Russell M E, Hancock W W, et al. Chronic rejection in experimental cardiac transplantation: studies in the Lewis-F344 model. Immunol Rev 1993; 134: 5-19.

34 Hayry $\mathrm{P}$, Isoniemi H, Yilmaz S, et al. Chronic allograft rejection. Immunol Rev 1993; 134: 33-81.

35 Gordon M, Luqmani R A, Adu D, et al. Relapses in patients with a systemic vasculitis. $Q \mathcal{F}$ Med 1993; 86: 779-89. 